\title{
NEAR-FIELD ASYMPTOTICS FOR THE POROUS MEDIUM EQUATION IN EXTERIOR DOMAINS. THE CRITICAL TWO-DIMENSIONAL CASE
}

\author{
C. CORTÁZAR, F. QUIRÓS AND N. WOLANSKI
}

\begin{abstract}
We consider the porous medium equation in an exterior twodimensional domain that excludes a hole, with zero Dirichlet data on its boundary. Gilding and Goncerzewicz proved in 2007 that in the far-field scale, which is the adequate one to describe the movement of the free boundary, solutions to this problem with integrable and compactly supported initial data behave as an instantaneous point-source solution for the equation with a variable mass that decays to 0 in a precise way, determined by the initial data and the hole. In this paper, starting from their result in the far field, we study the large time behavior in the near-field, in scales that evolve more slowly than the free boundary. In this way we get, in particular, the final profile and decay rate on compact sets. Spatial dimension two is critical for this problem, and involves logarithmic corrections.
\end{abstract}

\section{INTRODUCTION}

Let $\mathcal{H} \subset \mathbb{R}^{N}$ be a non-empty, bounded, simply connected open set with a $C^{2, \alpha}$ boundary. We assume, without loss of generality, that $0 \in \mathcal{H}$. Our aim is to determine the large time behavior of solutions to the Cauchy-Dirichlet problem for the porous medium equation in $\Omega:=\mathbb{R}^{N} \backslash \overline{\mathcal{H}}$ in the "near field" when $N=2$, by using the behavior in the "far field" found by Gilding and Goncerzewicz in [10]. More precisely, we are interested in the long time behavior of the solution $u$ to

(P) $\quad \partial_{t} u=\Delta u^{m}$ in $\Omega \times \mathbb{R}_{+}, \quad u=0$ in $\partial \mathcal{H} \times \mathbb{R}_{+}, \quad$ and $u(\cdot, 0)=u_{0}$ in $\Omega$,

with $m>1$, and nonnegative, compactly supported and integrable initial data $u_{0}$, in the critical case $N=2$. A full study of the asymptotic behavior for spatial dimensions $N \neq 2$ is already available [4, 8, 10, 12].

This problem, which models the flow of a fluid in a porous medium, does not have in general a classical solution, even if the initial data are smooth. Therefore, in the sequel we will deal with weak solutions.

Definition 1.1. A function $u$ is a weak ( $L^{1}$-energy) solution to Problem (P) if:

- $u \in C\left(\overline{\mathbb{R}_{+}}: L^{1}(\Omega)\right), u^{m} \in L_{\text {loc }}^{2}\left(\mathbb{R}_{+}: H_{0}^{1}(\Omega)\right)$;

- identity

$$
\int_{0}^{\infty} \int_{\Omega} u \partial_{t} \varphi d x d s-\int_{0}^{\infty} \int_{\Omega} \nabla u^{m} \cdot \nabla \varphi d x d s=0
$$

holds for every $\varphi \in C_{0}^{1}\left(\Omega \times \mathbb{R}_{+}\right)$;

2010 Mathematics Subject Classification. 35B40, 35K65, 35R35.

Key words and phrases. Porous medium equation, exterior domain, asymptotic behavior, matched asymptotics.

C. Cortázar supported by FONDECYT grant 1150028 (Chile). F. Quirós supported by project MTM2014-53037-P (Spain). N. Wolanski supported by CONICET PIP625, Res. 960/12, ANPCyT PICT-2012-0153, UBACYT X117 and MathAmSud 13MATH03 (Argentina). 
- $u(\cdot, 0)=u_{0}$ almost everywhere.

Existence of a weak solution follows from a double limit procedure, which is nowadays more or less standard, approximating first the initial datum by a sequence of bounded functions, and also approximating $\Omega$ by a sequence of bounded domains with null boundary data. The detailed proof involves a smoothing effect showing that weak solutions become immediately bounded. Uniqueness follows then, from a classical argument which combines the continuity in $L^{1}$ of the solution with an $L^{1}$ contraction property for bounded solutions. See [16] for similar arguments in the case in which the domain is the whole space. Let us finally mention that bounded weak solutions are continuous up to the boundary of the domain, [5, 18]. Therefore, due to the regularity of the solutions to uniformly parabolic equations, weak solutions to problem $(\mathrm{P})$ are classical in $\{u>0\}$ for all positive times.

The Cauchy problem. In the absence of holes, $\mathcal{H}=\emptyset$, the mass $M(t)=$ $\int_{\mathbb{R}^{N}} u(\cdot, t)$ of a solution to $(\mathrm{P})$ is conserved, no matter the spatial dimension, $M(t)=$ $\int_{\mathbb{R}^{N}} u_{0}$ for all $t \geq 0$. Moreover, as proved in [9], the solution behaves for large times as the instantaneous point-source solution of the equation with mass $M=\int_{\mathbb{R}^{N}} u_{0}$, that we denote by $\mathcal{U}(x, t ; M)$, in the following precise sense,

$$
\lim _{t \rightarrow \infty} t^{\frac{N}{N(m-1)+2}}\|u(\cdot, t)-\mathcal{U}(\cdot, t ; M)\|_{L^{\infty}\left(\mathbb{R}^{N}\right)}=0
$$

see also [15] and the references therein. This result does not require the initial data to be compactly supported. The special solution $\mathcal{U}(x, t ; M)$, which was discovered by Zel'dovič and Kompaneets [17] in dimensions one and three, and by Barenblatt [1] and Pattle [14] for arbitrary dimensions, has $M \delta$ (where $\delta$ is the Dirac distribution) as initial data. It has a selfsimilar form,

(1.2) $\mathcal{U}(x, t ; M)=t^{-\alpha} F_{M}(\xi), \quad$ where $\xi=\frac{x}{t^{\beta}}, \beta=\frac{1}{N(m-1)+2}$, and $\alpha=N \beta$,

with a profile

$$
\begin{aligned}
& F_{M}(\xi)=\left(\frac{(m-1) \beta}{2 m}\right)^{\frac{1}{m-1}}\left(\xi_{M}^{2}-|\xi|^{2}\right)_{+}^{\frac{1}{m-1}}, \text { where } \\
& \xi_{M}=\left(\frac{\Gamma\left(\frac{1}{2(m-1) \beta}\right)}{4 m \pi^{N / 2} \Gamma\left(\frac{m}{m-1}\right)}\right)^{(m-1) \beta}\left(\frac{2 m}{(m-1) \beta}\right)^{m \beta} M^{(m-1) \beta} .
\end{aligned}
$$

Notice that $\mathcal{U}(\cdot, t ; M)$ has a compact support for all times, namely $\left\{x \in \mathbb{R}^{N}\right.$ : $\left.|x| \leq \xi_{M} t^{\beta}\right\}$. This property, known as finite speed of propagation, is shared by all solutions having an integrable initial data with compact support.

Let us remark that the scaled variable

$$
w(\xi, \tau)=t^{\alpha} u\left(\xi t^{\beta}, t\right), \quad \text { where } t=\mathrm{e}^{\tau},
$$

satisfies the non-linear Fokker-Planck type equation

$$
\partial_{\tau} w(\xi, \tau)=\Delta w^{m}(\xi, \tau)+\beta \nabla \cdot(\xi w(\xi, \tau)),
$$

whose stationary (integrable) states are precisely the profiles $F_{M}$. When written in terms of this scaled variable, the convergence result (1.1) just says that $w$ converges uniformly as $t \rightarrow \infty(\tau \rightarrow \infty)$ towards a stationary state of (1.4), which one being dictated by the conservation of mass. 
A CONSERVATion LAW. In the presence of holes, solutions of $(\mathrm{P})$ do not conserve mass. However, we still have an invariant of the evolution, namely

$$
M_{\phi}(t):=\int_{\Omega} u(\cdot, t) \phi=\underbrace{\int_{\Omega} u_{0} \phi}_{M_{\phi}^{*}} \text { for all } t>0,
$$

where $\phi \in C(\bar{\Omega}) \cap C^{2}(\Omega)$ satisfies

$$
\Delta \phi=0 \text { in } \Omega \text { and } \phi=0 \text { on } \partial \Omega,
$$

plus some prescribed behavior at infinity; see [13] for a first proof of this fact in the radial case, and also [4] and [10]. In order to have a unique nontrivial solution to problem (1.6), not all behaviors at infinity are allowed. Which ones are possible depend on $N$. This will lead to different asymptotic behaviors for solutions to problem $(\mathrm{P})$ depending precisely on the dimension.

The PROBlem IN HIGH DIMENSIONS. When $N \geq 3$, in order to have a nontrivial solution to (1.6) we have to ask $\phi(x)$ to approach a constant as $|x| \rightarrow \infty$. This constant is chosen to be equal to one for simplicity. With this choice for $\phi$, the conservation law (1.5) yields that the mass $M(t):=\int_{\Omega} u(\cdot, t)$ converges to the nontrivial value $M_{\phi}^{*}$. Moreover, as proved in [4], the asymptotic behavior is given by

$$
\lim _{t \rightarrow \infty} t^{\alpha}\left\|u(\cdot, t)-\phi^{\frac{1}{m}} \mathcal{U}\left(\cdot, t ; M_{\phi}^{*}\right)\right\|_{L^{\infty}\left(\mathbb{R}^{N}\right)}=0 ;
$$

see also [11] for the linear case, $m=1$. Thus, in the far-field scale, $x=\xi t^{\beta}$ with $\xi \neq 0$, we have convergence towards the instantaneous point-source solution with mass $M_{\phi}^{*}$. More precisely, the outer behavior is given by

$$
t^{\alpha} u\left(\xi t^{\beta}, t\right) \rightarrow F_{M_{\phi}^{*}}(\xi) \text { uniformly for } 0<\xi_{1} \leq|\xi| \leq \xi_{2}<\infty,
$$

with $\alpha$ and $\beta$ as in (1.2); see also [10]. On the other hand, the behavior in the inner region, $|x|=o\left(t^{\beta}\right)$, is given in terms of the stationary solution,

$$
t^{\alpha} u(x, t)-F_{M_{\phi}^{*}}(0) \phi^{\frac{1}{m}}(x) \rightarrow 0 \quad \text { uniformly for }|x| \leq g(t) \text {, if } g(t)=o\left(t^{\beta}\right) .
$$

This includes the particular case of compact sets, which corresponds to $g(t)=C$.

Observe that for $x=g(t)$, with $g(t) \rightarrow \infty$ and $g(t)=o\left(t^{\beta}\right)$, which corresponds to $\xi \rightarrow 0$ in the outer scale, we have $t^{\alpha} u(x, t) \rightarrow F_{M_{\phi}^{*}}(0)$. This zone can be regarded both as the outer limit of the inner region and as the inner limit of the outer region. In fact, the coefficient multiplying $\phi^{1 / m}$ in the inner development is obtained from the outer one by matching both expansions in this common zone.

Let us remark that the rate of decay coincides, both in the inner and in the outer region, with that of the Cauchy problem. This is also true for the expansion rate; see the outer behavior. This is more clearly seen when studying the long time behavior of the support. Indeed, for compactly supported initial data, if we let

$$
\begin{aligned}
& \zeta_{+}(t)=\sup \{|x|: x \in \Omega \text { and } u(x, t)>0\}, \\
& \zeta_{-}(t)=\inf \{|x|: x \in \Omega \text { and } u(x, t)=0\},
\end{aligned}
$$

then, as proved in [4], $\zeta_{ \pm}(t) / t^{\beta} \rightarrow \xi_{M_{\phi}^{*}}$, where $\xi_{M}$ has the same meaning as in (1.3). An analogous result holds for the Cauchy problem; see for instance [15]. This means in particular that $u(x, t)$ is identically zero for $|x| \geq(1+\varepsilon) \xi_{M_{\phi}^{*}} t^{\beta}$ for all $\varepsilon>0$ and large times. This includes the very-far-field scale, $|x|=t^{\beta} g(t)$ with $g(t) \rightarrow \infty$. 
The PROBlem IN THE HALF-LINE. In dimension one, if there is a hole the mass goes to zero, $M(t)=O\left(t^{-1 /(2 m)}\right)$. Hence, we expect decay and expansion rates different from those of the Cauchy problem.

In this one-dimensional case, a hole disconnects the domain in several components, two of them unbounded. After a translation and maybe a reflection, the problem in these unbounded components can be transformed into the problem in the half-line $\mathbb{R}_{+}$. Nontrivial harmonic functions on the half-line with zero boundary values are multiples of $x$. Therefore, the conservation law means -in this case - that solutions have a constant first moment, and this fact indicates what is the right scaling in order to study the large time behavior: the one that preserves moment. Thus, solutions approach a so-called dipole solution $\mathcal{D}$ to the equation with the same first moment as $u_{0}$. More precisely, as proved in [12],

$$
\lim _{t \rightarrow \infty} t^{\frac{1}{m}}\left\|u(\cdot, t)-\mathcal{D}\left(\cdot, t ; M_{\phi}^{*}\right)\right\|_{L^{\infty}\left(\mathbb{R}_{+}\right)}=0, \quad \text { where } M_{\phi}^{*}=\int_{0}^{\infty} x u_{0}(x) d x .
$$

The special solution $\mathcal{D}$, discovered by Barenblatt and Zel'dovič [2], has a self-similar structure,

$$
\mathcal{D}(x, t ; M)=t^{-\alpha_{\mathrm{d}}} D_{M}(\xi), \quad \text { where } \xi=\frac{x}{t^{\beta_{\mathrm{d}}}}, \alpha_{\mathrm{d}}=\frac{1}{m}, \text { and } \beta_{\mathrm{d}}=\frac{1}{2 m},
$$

with a profile

$$
\begin{aligned}
& D_{M}(\xi)=\left(\frac{m-1}{2 m(m+1)}\right)^{\frac{1}{m-1}} \xi^{\frac{1}{m}}\left(\xi_{\mathrm{d}, M}^{\frac{m+1}{m}}-\xi^{\frac{m+1}{m}}\right)_{+}^{\frac{1}{m-1}}, \text { where } \\
& \xi_{\mathrm{d}, M}=\left(\frac{2 m(m+1)}{(m-1)\left(\int_{0}^{1} s^{\frac{m+1}{m}}\left(1-s^{\frac{m+1}{m}}\right)^{\frac{1}{m-1}} d s\right)^{m-1}}\right)^{\frac{1}{2 m}} M^{\frac{m-1}{2 m}},
\end{aligned}
$$

and, due to the choice of the similarity exponents $\alpha_{\mathrm{d}}$ and $\beta_{\mathrm{d}}$, its first moment is constant in time. Note that $\mathcal{D}$ has a compact support in space for all times.

Though the convergence result (1.7) is valid in the whole half-line, it only gives the exact decay rate and a nontrivial asymptotic profile in the far-field scale, $x=$ $\xi t^{\beta_{\mathrm{d}}}$ with $0<\xi<\xi_{\mathrm{d}, M_{\phi}^{*}}$, since $t^{\alpha_{\mathrm{d}}} \mathcal{D}\left(g(t), t ; M_{\phi}^{*}\right)=0$ if $g(t)=o\left(t^{\beta_{\mathrm{d}}}\right)$. The behavior in the very-far-field was also determined in [12], where the authors proved that $s(t)=\sup \{x: u(x, t)>0\}$ satisfies $s(t) / t^{\beta_{\mathrm{d}}} \rightarrow \xi_{\mathrm{d}, M_{\phi}^{*}}$.

As for the behavior in the near field, the dipole solution gives a hint of the right scaling. Indeed, the decay rate of $\mathcal{D}$ for $x=g(t)$ with $g(t)=o\left(t^{\beta_{\mathrm{d}}}\right)$ is $O\left(t^{-\alpha_{\mathrm{d}}-\beta_{\mathrm{d}} / m}(g(t))^{1 / m}\right)$. Having this in mind, we proved in [8], by a careful matching with the outer behavior, that

$$
\lim _{t \rightarrow \infty} t^{\alpha_{\mathrm{d}}+\frac{\beta_{\mathrm{d}}}{m}} \sup _{x \in \mathbb{R}_{+}} \frac{\left|u(x, t)-\mathcal{D}\left(x, t ; M_{\phi}^{*}\right)\right|}{(1+x)^{\frac{1}{m}}}=0,
$$

which settles the long time asymptotics in the near-field scale; see also [6] for the linear case. It may seem at first glance that stationary solutions do not play a role here. But they are still there, hidden in the dipole solution, since $\phi(\xi)=\xi$ and,

$$
D_{M}(\xi) \approx\left(\frac{m-1}{2 m(m+1)}\right)^{\frac{1}{m-1}} \xi_{\mathrm{d}, M}^{\frac{m+1}{m-1)}} \xi^{\frac{1}{m}} \quad \text { for } \xi \approx 0 .
$$

Thus, in particular,

$$
t^{\alpha_{\mathrm{d}}+\frac{\beta_{\mathrm{d}}}{m}} u(x, t) \rightarrow\left(\frac{m-1}{2 m(m+1)}\right)^{\frac{1}{m-1}} \xi_{\mathrm{d}, M_{\phi}^{*}}^{\frac{m+1}{m(m-1)}} x^{\frac{1}{m}} \quad \text { uniformly on compact sets. }
$$

Note that the decay rate, which differs from that of the Cauchy problem, depends on the scale, and is given by the ratio $x^{1 / m} / t^{\alpha_{\mathrm{d}}+\beta_{\mathrm{d}} / m}$. This makes the matching quite 
involved, since the overlapping region between the inner and the outer behavior is very narrow.

The CRITICAL CASE: OUter BEHAVIOR. In the critical two-dimensional case, the behavior at infinity leading to nontrivial stationary solutions is logarithmic. Thus, we have to look for $\phi \in C(\bar{\Omega}) \cap C^{2}(\Omega)$ satisfying

(S) $\quad \Delta \phi=0$ in $\Omega \subset \mathbb{R}^{2}, \quad \phi=0$ on $\partial \Omega$, and $|\phi(x)-\ln | x|| \leq C$ for all $x \in \bar{\Omega}$.

There is a unique such function if, for instance, $\mathcal{H} \in C^{2, \alpha}$ is simply connected. The conservation law (1.5) implies then, on the one hand, the global decay rate

$$
\|u(\cdot, t)\|_{L^{\infty}\left(\mathbb{R}^{2}\right)}=O\left((t \ln t)^{-\frac{1}{m}}\right) \quad \text { as } t \rightarrow \infty,
$$

and, on the other hand, that the mass satisfies

$$
\lim _{t \rightarrow \infty} \ln t M(t)=2 m M_{\phi}^{*} .
$$

Using these two facts, Gilding and Goncerzewicz proved that,

$$
\lim _{t \rightarrow \infty}(t \ln t)^{\frac{1}{m}} \sup _{x \in \mathcal{O}_{\delta}(t)}\left|u(x, t)-\mathcal{U}\left(x, t ; 2 m M_{\phi}^{*}(\ln t)^{-1}\right)\right|=0 \quad \text { for any } \delta>0,
$$

in outer sets

$$
\mathcal{O}_{\delta}(t)=\left\{x \in \Omega:|x| \geq \delta t^{\frac{1}{2 m}}(\ln t)^{-\frac{m-1}{2 m}}\right\} \quad \text { for } t \geq 1 .
$$

In addition, they also proved that

$$
t^{-\frac{1}{2 m}}(\ln t)^{\frac{m-1}{2 m}} \zeta_{ \pm}(t) \rightarrow \xi_{2 m M_{\phi}^{*}} \text { as } t \rightarrow \infty,
$$

where $\xi_{M}$ has the same meaning as in (1.3) (see [10] for these results).

It is worth noticing that the appropriate scaled function in this setting,

$$
w(\xi, \tau)=(t \ln t)^{\frac{1}{m}} u\left(\xi t^{\frac{1}{2 m}}(\ln t)^{-\frac{m-1}{2 m}}, t\right), \quad \text { where } t=\mathrm{e}^{\tau},
$$

does not satisfy the non-linear Fokker-Planck type equation (1.4), but a perturbation,

$$
\partial_{\tau} w(\xi, t)=\Delta w^{m}(\xi, t)+\frac{\nabla \cdot(\xi w(\xi, t))}{2 m}\left(1+\frac{1}{\tau}\right)-\frac{\xi \cdot \nabla w(\xi, \tau)}{2 \tau} .
$$

However, in the limit $t \rightarrow \infty(\tau \rightarrow \infty)$ the extra terms become negligible, and we have, also in this case, convergence towards a stationary solution to (1.4), which one being given by the conservation law (1.5).

Note that, though the function giving the asymptotic behavior in outer sets is not a solution of the equation, it still has a selfsimilar structure,

$$
\mathcal{U}\left(x, t ; 2 m M_{\phi}^{*}(\ln t)^{-1}\right)=(t \ln t)^{-\frac{1}{m}} F_{2 m M_{\phi}^{*}}(\tilde{\xi}), \text { where } \tilde{\xi}=x t^{-\frac{1}{2 m}}(\ln t)^{\frac{m-1}{2 m}}
$$

In comparison with the case with no holes, we find logarithmic corrections both in the decay and in the expansion rate.

The CRITICAL CASE: InNER BeHAVIOR. The aim of the present paper is to complement [10] by describing the behavior in inner sets of the form

$$
\mathcal{I}_{\delta}(t)=\left\{x \in \Omega:|x| \leq \delta t^{\frac{1}{2 m}}(\ln t)^{-\frac{m-1}{2 m}}\right\} \quad \text { for } t \geq 1 .
$$

This description is our main result. 
Theorem 1.1. Let $\mathcal{H} \ni 0$ be a simply connected, bounded, open subset of $\mathbb{R}^{2}$ with $C^{2, \alpha}$ boundary. If $u$ is a solution to $(\mathrm{P})$, for all $\delta \in\left(0, \delta_{*}\right)$, where $\delta_{*}=$ $\xi_{2 m M_{\phi}^{*}} \sqrt{(m-1) /(2 m)}$, there holds that

$$
\lim _{t \rightarrow \infty}\left(t \ln ^{2} t\right)^{\frac{1}{m}} \sup _{x \in \mathcal{I}_{\delta}(t)} \frac{\left|u(x, t)-\left(\frac{2 m \phi(x)}{\ln t}\right)^{\frac{1}{m}} \mathcal{U}\left(x, t ; 2 m M_{\phi}^{*}(\ln t)^{-1}\right)\right|}{(\ln (|x|+\mathrm{e}))^{\frac{1}{m}}}=0 .
$$

Note that the rate of decay is given by the ratio $\left(\ln |x| /\left(t \ln ^{2} t\right)\right)^{1 / m}$. Thus, there is a continuum of possible decay rates, starting with the decay rate $O\left((t \ln t)^{-1 / m}\right)$, holding in the far field, all the way up to $O\left(\left(t \ln ^{2} t\right)^{-1 / m}\right)$, that takes place on compact sets. Moreover, the scaled function $\left(t \ln ^{2} t / \ln (|x|+\mathrm{e})\right)^{1 / m} u(x, t)$ converges in the near-field scale, $|x| \leq t^{1 / 2 m} g(t)$ with $\lim _{t \rightarrow \infty} g(t)(\ln t)^{(m-1) / m}=0$, to a multiple of $(\phi(x) / \ln (|x|+\mathrm{e}))^{1 / m}$, where $\phi$ is the unique solution to $(\mathrm{S})$. In particular,

$$
\left(t \ln ^{2} t\right)^{\frac{1}{m}} u(x, t) \rightarrow F_{2 m M_{\phi}^{*}}(0)(2 m \phi(x))^{\frac{1}{m}} \quad \text { as } t \rightarrow \infty
$$

uniformly on compact sets.

Since $2 m \phi(x)(\ln t)^{-1}$ converges to 1 as time goes to infinity in regions where $|x|=O\left(t^{1 /(2 m)}(\ln t)^{-(m-1) / 2 m}\right)$, and taking also into account that the support grows as $O\left(t^{1 /(2 m)}(\ln t)^{-(m-1) / 2 m}\right)$, we can express the asymptotic behavior in all scales in a unified way.

Corollary 1.1. Under the hypotheses of Theorem 1.1

$$
\lim _{t \rightarrow \infty}\left(t \ln ^{2} t\right)^{\frac{1}{m}} \sup _{x \in \Omega} \frac{\left|u(x, t)-\left(\frac{2 m \phi(x)}{\ln t}\right)^{\frac{1}{m}} \mathcal{U}\left(x, t ; 2 m M_{\phi}^{*}(\ln t)^{-1}\right)\right|}{(\ln (|x|+\mathrm{e}))^{\frac{1}{m}}}=0 .
$$

This result is still valid in the linear case $m=1$, by taking $\mathcal{U}(x, t ; M)=$ $M \mathrm{e}^{-|x|^{2} /(4 t)}(4 \pi t)^{-1}$. This was proved in [11] by means of a representation formula for the solution of the problem in terms of the instantaneous point-source solution (the case of high dimensions, $N \geq 3$, was also treated there). In our non-linear setting, such a formula is not available, and we have to use an alternative approach, based on comparison with carefully chosen sub and supersolutions combined with a matching with the outer behavior. The matching is quite involved, since the rates of decay in inner and outer regions are not the same. We already had this difficulty in [7], where we dealt with a two-dimensional nonlocal linear heat equation.

Our main result holds even if $\mathcal{H}$ is not simply connected. But, for the sake of brevity we only prove it in this case. For general holes $\mathcal{H}$ Theorem 1.1 follows from a careful comparison with solutions of our problem with simply connected holes.

Organization OF THE PAPER. We devote Section 2 to the analysis of the stationary problem $(\mathrm{S})$. Convenient super and subsolutions with the adequate large time behavior are constructed in Sections 3 and 4, where we also get estimates leading to Theorem 1.1.

Notations. In what follows $B_{r}=\left\{x \in \mathbb{R}^{2}:|x|<r\right\}, F_{*}=F_{2 m M_{\phi}^{*}}, \xi_{*}=\xi_{2 m M_{\phi}^{*}}$, and $G(x, t)=\mathcal{U}\left(x, t ; 2 m M_{\phi}^{*}(\ln t)^{-1}\right)$.

\section{THE STATIONARY PROBLEM}

This section is devoted to studying the stationary problem $(\mathrm{S})$. Existence and uniqueness were already proved in [10]. 
Proposition 2.1 ([10], Lemma 2.1, based on [3], Proposition 4.10). Let $\mathcal{H}$ be a bounded, simply connected, open set with $C^{2, \alpha}$ boundary. There exists a unique harmonic function $\phi \in C^{2}(\Omega) \cap C(\bar{\Omega})$ such that $\phi=0$ on $\partial \mathcal{H}$ and $\phi(x)-\ln |x|=$ $o(\ln |x|)$ as $|x| \rightarrow \infty$.

Remark 2.1. The solution constructed in [10] actually satisfies that $|\phi(x)-\ln | x|| \leq$ $C$ in $\Omega$. In fact, for every $\varepsilon>0$ there is a radius $R_{\varepsilon}$ that we may take larger than $\varepsilon^{-1}$, such that $|\phi(x)-\ln | x|| \leq \varepsilon \ln |x|$ if $|x| \geq R_{\varepsilon}$. On the other hand, since $\mathcal{H}$ is bounded, there exists $C>0$ such that $\phi(x) \leq(1+\varepsilon) \ln |x|+C$ for $x \in \partial \mathcal{H}$. Therefore, $\phi(x) \leq(1+\varepsilon) \ln |x|+C$ for $x \in \Omega \cap B_{R_{\varepsilon}}$. Passing to the limit with $\varepsilon \rightarrow 0$ we obtain that $\phi(x) \leq \ln |x|+C$. The inequality $\phi(x) \geq \ln |x|-C$ is proved similarly.

In order to prove our main result, Theorem 1.1, we need $|\nabla \phi|$ to be comparable to $1 /|x|$. We start by checking that this is true "at infinity".

Proposition 2.2. Assume the hypotheses of Proposition 2.1. There exists $R>0$ such that the unique solution $\phi$ to $(\mathrm{S})$ satisfies

$$
\frac{1}{2} \leq x \cdot \nabla \phi(x) \leq|x||\nabla \phi(x)| \leq 2 \quad \text { for }|x| \geq R .
$$

Proof. Let $r>0$ such that $B_{2 r} \subset \mathcal{H}$. For $k \in \mathbb{N}$, let $\psi_{k}(x)=\phi(k x)-\ln (k|x| / r)$. Then, $\psi_{k}$ is harmonic in $\mathbb{R}^{2} \backslash \overline{\mathcal{H}}_{k}$ where $\mathcal{H}_{k}=\left\{x \in \mathbb{R}^{2}: k x \in \mathcal{H}\right\}$. Moreover, there exists $C>0$ such that $\left|\psi_{k}(x)\right| \leq C$ in $\mathbb{R}^{2} \backslash \overline{\mathcal{H}}_{k}$. Hence, for every sequence $\left\{k_{n}\right\}$ with $k_{n} \rightarrow \infty$ there is a subsequence $\left\{k_{n_{j}}\right\}$ and a harmonic and bounded function $\psi$ such that $\psi_{k_{n_{j}}} \rightarrow \psi$ as $j \rightarrow \infty$ uniformly on compact subsets of $\mathbb{R}^{2} \backslash\{0\}$. Since $\psi$ is harmonic and bounded in $\mathbb{R}^{2} \backslash\{0\}$, it can be extended to a harmonic and bounded function in $\mathbb{R}^{2}$ that, by Liouville's Theorem, is constant: $\psi \equiv C_{0}$.

Let $\zeta_{j}=\psi_{k_{n_{j}}}-C_{0}$. Since $\zeta_{j} \rightarrow 0$ uniformly on compact subsets of $\mathbb{R}^{2} \backslash\{0\}$, then $\left|\nabla \zeta_{j}\right| \rightarrow 0$ uniformly in $B_{2} \backslash B_{\frac{1}{2}}$. Since the limit is independent of the subsequence, convergence is not restricted to subsequences, and hence

$$
k \nabla \phi(k x) \rightarrow \frac{x}{|x|^{2}} \quad \text { uniformly for } \frac{1}{2} \leq|x| \leq 2 \text { as } k \rightarrow \infty .
$$

Therefore, taking $y=k x$ with $|x|=1$, which means $k=|y|$, we conclude that

$$
|y| \nabla \phi(y) \rightarrow \frac{y}{|y|} \quad \text { as }|y| \rightarrow \infty .
$$

Estimate (2.1) now follows easily by observing that

$$
x \cdot \nabla \phi(x)=1+\frac{x}{|x|} \cdot\left(|x| \nabla \phi(x)-\frac{x}{|x|}\right),
$$

since we know from (2.2) that $\left.|| x|\nabla \phi(x)-x| x\right|^{-1} \mid \leq 1 / 2$ if $|x| \geq R$ for some large $R$.

Since $\partial \mathcal{H} \in C^{2, \alpha}$, then $\phi \in C^{2}(\bar{\Omega})$, and we deduce that $|x||\nabla \phi(x)| \leq C$ in $\bar{\Omega}$. In order to proceed further, we still have to prove that $|x||\nabla \phi(x)| \geq c>0$ in bounded sets. Since $|\nabla \phi| \geq c_{0}>0$ in a neighborhood of $\partial \mathcal{H}$, we only need to check that $\nabla \phi \neq 0$ in $\Omega$. This is indeed the case as we prove next.

Proposition 2.3. Let $\mathcal{H} \ni 0$ be a bounded, simply connected, open subset of $\mathbb{R}^{2}$ with $C^{2, \alpha}$ boundary. Let $\phi$ be the unique solution to (S). Then, $\nabla \phi \neq 0$ in $\bar{\Omega}$. 
Proof. We will get a semi-explicit formula for the solution $\phi$, from where the result follows immediately.

Let $r>0$ be such that $B_{r} \subset \mathcal{H}$. First, we perform an inversion with respect to $B_{r}$. The "inverted" set

$$
\Omega^{\prime}=\left\{x=\frac{r^{2} y}{|y|^{2}}: y \in \Omega\right\} \cup\{0\}
$$

is a simply connected open set contained in $B_{r}$. Moreover, since $\partial \mathcal{H}$ is a Jordan arc and $0 \notin \partial \mathcal{H}$, then

$$
\partial \Omega^{\prime}=\left\{x=\frac{r^{2} y}{|y|^{2}}: y \in \partial \mathcal{H}\right\}
$$

is also a Jordan arc. The Kelvin transform $\psi(x)=\phi\left(r^{2} x|x|^{-2}\right)$ is well defined and harmonic in $\Omega^{\prime} \backslash\{0\}, \psi=0$ on $\partial \Omega^{\prime}$, and $\psi(x)-\ln (1 /|x|)$ is bounded.

Let now $f: B_{1} \rightarrow \Omega^{\prime}$ be a conformal mapping. This is, $f$ is holomorphic in $B_{1}$, continuous in $\bar{B}_{1}, f\left(\partial B_{1}\right)=\partial \Omega^{\prime}, f$ is one to one between $B_{1}$ and $\Omega^{\prime}, f^{\prime}(x) \neq 0$ for $x \in B_{1}, f(0)=0$ and $\mathfrak{a}:=f^{\prime}(0) \in \mathbb{R}_{+}$. The existence (and uniqueness) of such a mapping is guaranteed by Riemann's Mapping Theorem. Let

$$
\varphi(x)=\psi(f(x))=\phi\left(\frac{r^{2} f(x)}{|f(x)|^{2}}\right) \text { for } x \in B_{1} \backslash\{0\} .
$$

Since $f(0)=0$ and $\mathfrak{a}=f^{\prime}(0)>0$, there exists $\varepsilon>0$ such that $2 \mathfrak{a}|x| \geq|f(x)| \geq$ $(\mathfrak{a} / 2)|x|$ if $|x| \leq \varepsilon$. Thus, since $f$ is one to one, there exists $\delta>0$ such that $\delta<|f(x)|<r$ if $\varepsilon<|x|<1$. Hence,

$$
\left|\ln \frac{1}{|f(x)|}-\ln \frac{1}{\mathfrak{a}|x|}\right|=\left|\ln \frac{|f(x)|}{\mathfrak{a}|x|}\right| \leq C \quad \text { for } 0<|x|<1 .
$$

On the other hand, for $x \in B_{1} \backslash\{0\}$ and $y=r^{2} f(x)|f(x)|^{-2} \in \bar{\Omega}$,

$$
\left|\varphi(x)-\ln \frac{1}{|f(x)|}\right|=\left|\phi\left(\frac{r^{2} f(x)}{|f(x)|^{2}}\right)-\ln \frac{1}{|f(x)|}\right|=|\phi(y)-\ln | y|+2 \ln r|,
$$

and hence, from (2.3) and (2.4) we deduce that $\varphi(x)-\ln (1 /|x|)$ is bounded in $B_{1} \backslash\{0\}$. Therefore, since $\varphi$ is harmonic in $B_{1} \backslash\{0\}$ and $\varphi=0$ on $\partial B_{1}$, we deduce that $\varphi(x)-\ln (1 /|x|)=0$ in $B_{1} \backslash\{0\}$. Thus, $\phi(x)=-\ln \left|f^{-1}\left(r^{2} x /|x|^{2}\right)\right|$. In particular, $\nabla \phi \neq 0$ in $\bar{\Omega}$.

Corollary 2.1. Under the hypotheses of Proposition 2.3, there exist constants $C, c>0$ such that

$$
c \leq|x||\nabla \phi(x)| \leq C \quad \text { in } \bar{\Omega} .
$$

\section{Control from above}

In this section we construct a suitable strict classical supersolution $V$ of the porous medium equation in the inner region

$$
\mathcal{I}_{\delta, T}=\left\{(x, t): x \in \mathcal{I}_{\delta}(t), t \geq T\right\}=\left\{(x, t): x \in \Omega,|x| \leq \delta t^{\frac{1}{2 m}}(\ln t)^{-\frac{m-1}{2 m}}, t \geq T\right\},
$$

with $\delta>0$ small enough and $T$ big enough, and we use it to prove the "upper part" of Theorem 1.1.

Definition 3.1. A strict classical supersolution (resp. subsolution) of the porous medium equation in $\mathcal{I}_{\delta, T}$ is a function $V$ (resp. $\left.v\right) \in C\left(\overline{\mathcal{I}_{\delta, T}}\right) \cap C_{x, t}^{2,1}\left(\operatorname{Int}\left(\mathcal{I}_{\delta, T}\right)\right)$ that satisfies $V_{t}-\Delta V^{m}>0$ (resp. $v_{t}-\Delta v^{m}<0$ ) in $\operatorname{Int}\left(\mathcal{I}_{\delta, T}\right)$. 
Remark 3.1. If $u$ is the weak solution of $(\mathrm{P})$, then $u$ is continuous in $\bar{\Omega} \times \mathbb{R}_{+}$and, -due to (1.11) - if $\delta>0$ is small enough and $T$ is large enough, there holds that $u>0$ in $\mathcal{I}_{\delta, T}$ and $u$ is a classical solution in its interior.

We will construct $V$ so that, if $\delta>0$ is small and $T$ is large we have, $V>u$ on the parabolic boundary:

$$
\begin{aligned}
\partial_{p} \mathcal{I}_{\delta, T} & :=\left\{(x, T): x \in \Omega,|x| \leq \delta T^{\frac{1}{2 m}}(\ln T)^{-\frac{m-1}{2 m}}\right\} \\
& \cup\left\{(x, t): x \in \Omega,|x|=\delta t^{\frac{1}{2 m}}(\ln t)^{-\frac{m-1}{2 m}}, t \geq T\right\} \cup\{x \in \partial \mathcal{H}, t \geq T\} .
\end{aligned}
$$

This is enough to get that $V>u$ in $\mathcal{I}_{\delta, T}$, as we prove next.

Lemma 3.1. If $u$ is the weak solution of $(\mathrm{P}), \delta$ is small enough and $T$ is large enough, and if $V$ (resp. $v$ ) is a strict classical supersolution (resp. subsolution) in $\mathcal{I}_{\delta, T}$ such that $V>u($ resp. $v<u)$ on $\partial_{p} \mathcal{I}_{\delta, T}$, then $V>u($ resp. $v<u)$ in $\mathcal{I}_{\delta, T}$.

Proof. It follows from a standard parabolic argument. We prove it in the case of the supersolution. The case of the subsolution is proved similarly.

Suppose it is not so, then, as $u$ and $V$ are continuous, there is a first time $t_{0}$ and a point $x_{0}$ such that $\left(x_{0}, t_{0}\right) \in \mathcal{I}_{\delta, T}^{\circ}, u<V$ for $T \leq t<t_{0}, u\left(x_{0}, t_{0}\right)=V\left(x_{0}, t_{0}\right)$, and $u\left(x, t_{0}\right) \leq V\left(x, t_{0}\right)$. At such point $u_{t} \geq V_{t}$ and $\Delta u^{m} \leq \Delta V^{m}$, which is a contradiction.

We want $V$ to be a good approximation of the asymptotic limit $\tilde{V}(x, t)=$ $G(x, t)\left(2 m \phi(x)(\ln t)^{-1}\right)^{1 / m}$. Thus, we define

$$
V(x, t)=\eta c(t) G(x, t) W(x, t),
$$

where

$$
\begin{aligned}
& \eta>1, c(t)=1+\kappa_{0}\left(\frac{T}{t}\right)^{\mu} \text { for } \kappa_{0}>0 \text { and } \mu \in(0,1), \text { and } \\
& \left.W(x, t)=\left(\frac{2 m}{\ln t}\right)^{\frac{\nu(t)}{m}}\left((\phi(x))^{\nu(t)}+k\right)\right)^{\frac{1}{m}} \text { for } k>0, \text { with } \\
& \nu(t)=1-\frac{1}{\ln t} .
\end{aligned}
$$

The values of $\eta$ and $k$ will be chosen so that $V>u$ on the lateral boundary, $c(t)$ so that $V>u$ on $\left\{(x, T): x \in \Omega,|x| \leq \delta T^{1 /(2 m)}(\ln T)^{-(m-1) / m}\right\}$ and $\nu(t)$ so that $\partial_{t} V>\Delta V^{m}$ in $\mathcal{I}_{\delta, T}^{\circ}$.

We note that $\partial_{t} V-\Delta V^{m}=\mathcal{A}+\mathcal{B}$, where

$$
\begin{aligned}
& \mathcal{A}=\eta c^{\prime} G w+\eta c W \partial_{t} G-\eta^{m} c^{m} W^{m} \Delta G^{m}+\eta c G \partial_{t} W, \\
& \mathcal{B}=-\eta^{m} c^{m} G^{m} \Delta W^{m}-2 \eta^{m} c^{m} \nabla W^{m} \cdot \nabla G^{m} .
\end{aligned}
$$

We will see that both $\mathcal{A}$ and $\mathcal{B}$ are nonnegative in the outer part of the inner region given by

$$
\mathcal{I}_{\delta, T}^{\mathrm{O}}=\left\{(x, t) \in \mathcal{I}_{\delta, T}:|x| \geq t^{\frac{1}{2 m}}(\ln t)^{-2}\right\} .
$$

However, though $\mathcal{B}$ is still positive in the inner part of the inner region

$$
\mathcal{I}_{\delta, T}^{\mathrm{i}}=\left\{(x, t) \in \mathcal{I}_{\delta, T}:|x| \leq t^{\frac{1}{2 m}}(\ln t)^{-2}\right\},
$$

this is not necessarily the case for $\mathcal{A}$. Hence, we will need to estimate carefully both terms to check that, anyway, $\mathcal{A}+\mathcal{B}$ is positive in this set.

Remark 3.2. We can take any power larger than 2 for the logarithmic correction in the definition of $\mathcal{I}_{\delta, T}^{\mathrm{O}}$ and $\mathcal{I}_{\delta, T}^{\mathrm{i}}$. 
Lemma 3.2. Let $\mathcal{A}$ be defined by (3.2)-(3.3) and $\delta_{*}:=\xi_{*} \sqrt{(m-1) /(2 m)}$. There are a constant $\gamma=\gamma\left(m, M_{\phi}^{*}, k\right)>0$ and a time $T_{\mathcal{A}}=T_{\mathcal{A}}\left(\Omega, m, M_{\phi}^{*}, k\right)$ such that

$$
\mathcal{A}(x, t)>-\frac{\gamma \eta c(t)}{t^{1+\frac{1}{m}}(\ln t)^{\frac{1}{m}}} \quad \text { in } \mathcal{I}_{\delta, T} \text { for any } \delta \in\left(0, \delta_{*}\right) \text { and } T \geq T_{\mathcal{A}}
$$

Moreover, there are values $T_{\mathcal{A}}^{\mathrm{o}}=T_{\mathcal{A}}^{\mathrm{o}}\left(\eta, \Omega, m, M_{\phi}^{*}, k\right)$ and $\mu_{*}=\mu_{*}\left(\eta, m, M_{\phi}^{*}\right)>0$ such that $\mathcal{A}>0$ in $\mathcal{I}_{\delta, T}^{\mathrm{O}}$ for all $\delta \in\left(0, \delta_{*}\right)$ and $\mu \in\left(0, \mu_{*}\right)$ if $T \geq T_{\mathcal{A}}^{\mathrm{o}}$.

Proof. Straightforward computations show that, for any $\delta \in\left(0, \xi_{*}\right)$ and $T \geq \mathrm{e}$,

$$
\begin{aligned}
& t c^{\prime}(t) \geq-\mu c(t) \\
& \partial_{t} G(x, t)=\Delta G^{m}(x, t)-\frac{\left(2 m M_{\phi}^{*}\right)^{\frac{1}{m}}}{(t \ln t)^{1+\frac{1}{m}}}\left(1-\frac{|\tilde{\xi}|^{2}}{\xi_{*}^{2}}\right)^{\frac{1}{m-1}-1}, \\
& \Delta G^{m}(x, t)=-\frac{1}{m t^{1+\frac{1}{m}}(\ln t)^{\frac{1}{m}}}\left(\frac{M_{\phi}^{*}}{2 \pi}\right)^{\frac{1}{m}}\left(1-\frac{|\tilde{\xi}|^{2}}{\xi_{*}^{2}}\right)^{\frac{1}{m-1}-1}\left(1-\frac{m}{m-1} \frac{|\tilde{\xi}|^{2}}{\xi_{*}^{2}}\right) \\
& \partial_{t} W(x, t)=\frac{W(x, t)}{m}\left(\frac{(\phi(x))^{\nu(t)} \ln \phi(x)}{\left((\phi(x))^{\nu(t)}+k\right) t(\ln t)^{2}}-\frac{\ln \left(\frac{\ln t}{2 m}\right)}{t(\ln t)^{2}}-\frac{\nu(t)}{t \ln t}\right)
\end{aligned}
$$

in $\mathcal{I}_{\delta, T}$.

Moreover, there is a time $T_{w}=T_{w}(\Omega, m, k)$ such that $w \leq 2$ in $\mathcal{I}_{\delta, T}$ if $T \geq T_{w}$. Thus, there are positive constants $\gamma_{1}=\gamma_{1}\left(m, M_{\phi}^{*}, k\right)$ and $\gamma_{2}=\gamma_{2}\left(m, M_{\phi}^{*}\right)$ such that

$$
\partial_{t} W(x, t)>-\frac{\gamma_{1}}{t \ln t} \quad \text { and } 0>\Delta G^{m}(x, t)>-\frac{\gamma_{2}}{t^{1+\frac{1}{m}}(\ln t)^{\frac{1}{m}}} \quad \text { in } \mathcal{I}_{\delta, T} \text {, }
$$

for any $\delta \in\left(0, \delta_{*}\right)$ and $T \geq T_{w}$. Since $0 \leq G(x, t)<F_{*}(0) /(t \ln t)^{1 / m}$, we easily get (3.4) if $T \geq T_{\mathcal{A}} \geq T_{w}$, with $T_{\mathcal{A}}=T_{\mathcal{A}}\left(\Omega, m, M_{\phi}^{*}, k\right)$ large enough.

In order to prove the positivity of $\mathcal{A}$ in $\mathcal{I}_{\delta, T}^{\mathrm{O}}$ we will show that the good term $-\eta^{m} c^{m} W^{m} \Delta G^{m}$ is not only positive there, but also big enough to compensate for the negative leading order terms, if $\mu$ is small. Indeed, since $\phi(x) \geq \ln |x|-C$ and

$$
\ln |x| \geq \frac{\ln t}{2 m}-2 \ln \ln t \quad \text { in } \mathcal{I}_{\delta, T}^{\mathrm{O}}
$$

there exists a time $T_{\eta, m}$ depending only on $\eta$ and $m$ such that

$$
(\eta W(x, t))^{m-1} \geq 1+\frac{\eta^{m-1}-1}{2} \quad \text { for } t \geq T_{\eta, m}
$$

Hence, taking $T \geq \max \left(T_{\mathcal{A}}, T_{\eta, m}\right)$, since $c(t)>1$ for every $t \geq T$, we get

$$
\mathcal{A}(x, t) \geq \frac{\eta c(t) W(x, t)}{t^{1+\frac{1}{m}}(\ln t)^{\frac{1}{m}}}\left(\frac{\gamma_{2}}{2}\left(\eta^{m-1}-1\right)-\mu F_{*}(0)+o(1)\right) \quad \text { in } \mathcal{I}_{\delta, T}^{\mathrm{O}} .
$$

The result follows just by taking $\mu_{*}=\gamma_{2}\left(\eta^{m-1}-1\right) /\left(4 F_{*}(0)\right)$, and then $T_{\mathcal{A}}^{\mathrm{o}} \geq$ $\max \left(T_{\mathcal{A}}, T_{\eta, m}\right)$ big enough.

Lemma 3.3. Let $\mathcal{B}$ be defined by $(3.2)-(3.3)$. There is a time $T_{\mathcal{B}}^{\mathrm{o}}=T_{\mathcal{B}}^{\mathrm{o}}(\Omega, m)$ such that $\mathcal{B}>0$ in $\mathcal{I}_{\delta, T}^{\mathrm{O}}(s)$ for all $T \geq T_{\mathcal{B}}^{\mathrm{O}}$ and $\delta \in\left(0, \xi_{*}\right)$. Moreover, there are a constant $\varpi=\varpi\left(m, M_{\phi}^{*}\right)$ and a time $T_{\mathcal{B}}^{\mathrm{i}}=T_{\mathcal{B}}^{\mathrm{i}}\left(m, M_{\phi}^{*}\right)$ such that for all $\delta \in\left(0, \xi_{*}\right)$ and $T \geq T_{\mathcal{B}}^{\mathrm{i}}$,

$$
\mathcal{B}(x, t) \geq \frac{\varpi \eta c(t)}{t^{1+\frac{1}{m}}} \quad \text { in } \mathcal{I}_{\delta, T}^{\mathrm{i}}
$$


Proof. Easy computations yield, for any $\delta \in\left(0, \xi_{*}\right)$ and $(x, t) \in \mathcal{I}_{\delta, T}$,

$$
\begin{aligned}
& \nabla G^{m}(x, t)=-\frac{1}{2 m t^{1+\frac{1}{m}}(\ln t)^{\frac{1}{m}}}\left(\frac{M_{\phi}^{*}}{2 \pi}\right)^{\frac{1}{m}}\left(1-\frac{|\tilde{\xi}|^{2}}{\xi_{*}^{2}}\right)^{\frac{1}{m-1}} x, \\
& \nabla W^{m}(x, t)=\nu(t)\left(\frac{2 m}{\ln t}\right)^{\nu(t)}(\phi(x))^{\nu(t)-1} \nabla \phi(x), \text { and } \\
& \Delta W^{m}(x, t)=\nu(t)(\nu(t)-1)\left(\frac{2 m}{\ln t}\right)^{\nu(t)}(\phi(x))^{\nu(t)-2}|\nabla \phi(x)|^{2} .
\end{aligned}
$$

Moreover, given $R$ as in estimate $(2.1)$, there is a time $T_{\mathcal{B}}^{\mathrm{o}}=T_{\mathcal{B}}^{\mathrm{o}}(\Omega, m)$ such that $|x|>R$ in $\mathcal{I}_{\delta, T}^{\mathrm{O}}$ for any $T \geq T_{\mathcal{B}}^{\mathrm{O}}$, which immediately implies, by (2.1), that $\nabla W^{m} \cdot \nabla G^{m}<0$ there. On the other hand, $\Delta W^{m}<0$, since $\nu(t)<1$, and hence $\mathcal{B}>0$.

In order to estimate the behavior in the inner part of the inner region, we notice that there is a time $T_{\phi}=T_{\phi}\left(m, M_{\phi}^{*}\right)$ such that $|\tilde{\xi}|<\xi_{*} / 2, \phi(x) \leq(\ln t) / m$ and $1 / 2 \leq \nu(t)<1$ in $\mathcal{I}_{\delta, T}^{\text {i }}$ if $T \geq T_{\phi}$. Hence, using also that $|\nabla \phi(x)|$ is comparable to $1 /|x|$ in $\Omega$, we easily get that there are positive constants $\varpi_{1}=\varpi_{1}\left(m, M_{\phi}^{*}\right)$ and $\varpi_{2}=\varpi_{2}\left(m, M_{\phi}^{*}\right)$ such that

$$
-\left(G^{m} \Delta W^{m}\right)(x, t) \geq \frac{\varpi_{1}}{t^{1+\frac{1}{m}}} \quad \text { and }-2\left(\nabla G^{m} \cdot \nabla W^{m}\right)(x, t) \geq-\frac{\varpi_{2}}{(t \ln t)^{1+\frac{1}{m}}}
$$

in $\mathcal{I}_{\delta, T}^{\mathrm{i}}$, if $\delta \in\left(0, \xi_{*}\right)$ and $T \geq T_{\phi}$. Since $\eta, c(t)>1$, we finally get $(3.7)$ if $T \geq T_{\mathcal{B}}^{\mathrm{i}} \geq$ $T_{\phi}$, with $T_{\mathcal{B}}^{\mathrm{i}}=T_{\mathcal{B}}^{\mathrm{i}}\left(m, M_{\phi}^{*}\right)$ large enough.

Combining the estimates in Lemmas 3.2 and 3.3, we immediately get that $V$ is a strict classical supersolution of the equation. Note that $\delta_{*}<\xi_{*}$.

Corollary 3.1. Let $V$ be defined by (3.2) and $\delta_{*}$ and $\mu_{*}$ as in Lemma 3.2. There is a time $T_{*}=T_{*}\left(\eta, \Omega, m, M_{\phi}^{*}, k\right)$ such that

$$
\partial_{t} V-\Delta V^{m}>0 \quad \text { in } \mathcal{I}_{\delta, T} \text { for all } \delta \in\left(0, \delta_{*}\right), \mu \in\left(0, \mu_{*}\right) \text {, and } T \geq T_{*} \text {. }
$$

In order to prove that $u<V$ in $I_{\delta, T}$, it is then just enough to show that this inequality holds in the parabolic boundary of the set. The ordering in the outer boundary will come from the far-field behavior. It is here where we are performing the matching.

Proposition 3.1. Let $u$ be a solution to $(\mathrm{P}), \eta>1, V$ as in $(3.2)$, and $\delta_{*}$ and $\mu_{*}$ as in Lemma 3.2. Given $k>0$ and $\delta \in\left(0, \delta_{*}\right)$, there is a time $T^{+}=$ $T^{+}\left(\eta, \delta, \Omega, m, M_{\phi}^{*}, k\right)>0$ such that for any $T \geq T^{+}$there is a value $\kappa_{0}>0$ so that, for all $\mu \in\left(0, \mu_{*}\right)$, there holds that $u<V$ in $\mathcal{I}_{\delta, T}$.

Proof. We first note that $u=0<V$ on $\partial \Omega \times \mathbb{R}_{+}$. As for the outer boundary, we have from (1.10) that there exists $T_{\eta, \delta, m}>0$ such that, if $|x|=\delta t^{1 /(2 m)}(\ln t)^{-(m-1) / 2 m}$ with $t \geq T_{\eta, \delta, m}$, then

$$
u(x, t) \leq G(x, t)+\frac{(\eta-1) F_{*}\left(\delta_{*}\right)}{2(t \ln t)^{\frac{1}{m}}} \leq\left(1+\frac{\eta-1}{2}\right) G(x, t) .
$$

On the other hand, there is a time $T_{\Omega, \eta, \delta}>0$ such that

$$
\eta W(x, t) \geq 1+\frac{\eta-1}{2} \text { for }|x|=\delta t^{\frac{1}{2 m}}(\ln t)^{-\frac{m-1}{2 m}}, \text { with } t \geq T_{\Omega, \eta, \delta} .
$$

Since $c(t)>1$, we conclude that

$$
u(x, t)<V(x, t) \quad \text { for }|x|=\delta t^{\frac{1}{2 m}}(\ln t)^{-\frac{m-1}{2 m}} \text {, with } t \geq \max \left(T_{\eta, \delta, m}, T_{\Omega, \eta, \delta}\right) .
$$


Finally, given $T \geq T^{+}:=\max \left(T_{*}, T_{\eta, \delta, m}, T_{\Omega, \eta, \delta}\right)$, where $T_{*}$ is the time given by Corollary 3.1 we have,

$$
V(x, T) \geq \frac{\left(1+\kappa_{0}\right) k^{\frac{1}{m}} F_{*}\left(\delta_{*}\right)}{(T \ln T)^{\frac{1}{m}}\left(\frac{\ln T}{2 m}\right)^{\frac{\nu(T)}{m}}}>\left\|u_{0}\right\|_{L^{\infty}} \geq u(x, T),
$$

if $\kappa_{0}>0$ is big enough. The result then follows from Lemma 3.1.

The "upper part" of Theorem 1.1 now follows immediately.

Theorem 3.1. Assume the hypotheses of Theorem 1.1. Given $\varepsilon>0$, there exists a time $T_{\varepsilon}$ such that, for all $\delta \in\left(0, \delta_{*}\right)$,

$$
\mathcal{M}(x, t):=t^{\frac{1}{m}}(\ln t)^{\frac{2}{m}} \frac{\left(u(x, t)-\left(\frac{2 m \phi(x)}{\ln t}\right)^{\frac{1}{m}} G(x, t)\right)}{(\ln (|x|+\mathrm{e}))^{\frac{1}{m}}} \leq \varepsilon \quad \text { in } \mathcal{I}_{\delta, T_{\varepsilon}} .
$$

Proof. We decompose $\mathcal{M}$ as

$$
\begin{aligned}
\mathcal{M}(x, t) & =\frac{t^{\frac{1}{m}}(\ln t)^{\frac{2}{m}}}{(\ln (|x|+\mathrm{e}))^{\frac{1}{m}}}((u(x, t)-c(t) W(x, t) G(x, t)) \\
& +(c(t)-1) W(x, t) G(x, t)+\left(W(x, t)-\left(\frac{2 m \phi(x)}{\ln t}\right)^{\frac{\nu(t)}{m}}\right) G(x, t) \\
& \left.+\left(\frac{2 m \phi(x)}{\ln t}\right)^{\frac{1}{m}}\left(\left(\frac{2 m \phi(x)}{\ln t}\right)^{\frac{\nu(t)-1}{m}}-1\right) G(x, t)\right) .
\end{aligned}
$$

Let $\eta=1+\varepsilon, k=\varepsilon^{m}$, and $\delta \in\left(0, \delta_{*}\right)$. By Proposition 3.1, given any $T \geq T^{+}$, there are values $\kappa_{0}>0$ and $\mu \in\left(0, \mu_{*}\right)$ such that

$$
u(x, t)-c(t) W(x, t) G(x, t) \leq \varepsilon c(t) W(x, t) G(x, t) \quad \text { if }(x, t) \in \mathcal{I}_{\delta, T} .
$$

On the other hand, since $(a+b)^{1 / m}-a^{1 / m} \leq b^{1 / m}$ for all $a, b>0$ if $m>1$, we have

$$
W(x, t)-\left(\frac{2 m \phi(x)}{\ln t}\right)^{\frac{\nu(t)}{m}} \leq\left(\frac{2 m}{\ln t}\right)^{\frac{\nu(t)}{m}} \varepsilon .
$$

We finally observe that $(\ln t)^{(\nu(t)-1) / m} \rightarrow 1$ as $t \rightarrow \infty$ so that, since $\phi(x) \leq \ln |x|+C$ and $G(x, t) \leq(t \ln t)^{-1 / m} F_{*}(0)$,

$$
0 \leq \frac{t^{\frac{1}{m}}(\ln t)^{\frac{2}{m}}}{\left(\ln (|x|+\mathrm{e})^{\frac{1}{m}}\right.} w(x, t) G(x, t) \leq C \quad \text { for }(x, t) \in \mathcal{I}_{\delta, T},
$$

Therefore, using also that $c(t) \rightarrow 1$, we conclude that there is a time $T_{\varepsilon} \geq T^{+}$such that $\mathcal{M}(x, t) \leq K \varepsilon$ for all $t \geq T_{\varepsilon}$ for some constant $K$ independent of $\varepsilon$.

\section{Control from Below}

In this section we prove the "lower part" of Theorem 1.1. The construction of the subsolution is a bit more involved than that of the supersolution. Comparison will be performed in the intersection of the inner set $\mathcal{I}_{\delta, T}$ with an approximation of $\Omega$. So, let us start by constructing this approximate set.

Let $r_{0}$ be small so that, on the one hand, the set $\mathcal{N}_{2 r_{0}}=\{x \in \bar{\Omega}: \operatorname{dist}\{x, \mathcal{H}\} \leq$ $\left.2 r_{0}\right\}$ can be parametrized by $x=\bar{x}+s \mathbf{n}(\bar{x}), \bar{x} \in \partial \mathcal{H}, 0 \leq s \leq 2 r_{0}$, where $\mathbf{n}(\bar{x})$ is the exterior unit normal to $\partial \mathcal{H}$ at $\bar{x}$, and on the other hand $\phi(\bar{x}+s \mathbf{n}(\bar{x})) \geq \kappa s$ in $\mathcal{N}_{2 r_{0}}$ with $\kappa>0$. Notice that

$$
\Gamma_{0}:=\left\{x=\bar{x}+r_{0} \mathbf{n}(\bar{x}): \bar{x} \in \partial \mathcal{H}\right\}=\left\{x \in \bar{\Omega}: \operatorname{dist}\{x, \mathcal{H}\}=r_{0}\right\}
$$

is a $C^{1, \alpha}$ surface. Let $\bar{\alpha}_{0}=\inf _{\Gamma_{0}} \phi(x)$. Since $\phi(x) \rightarrow \infty$ as $|x| \rightarrow \infty$ and $\phi$ is harmonic in $\Omega$, then $\phi(x) \geq \bar{\alpha}_{0}$ if dist $\{x, \mathcal{H}\} \geq r_{0}$. 
Let now $\alpha_{0} \in\left(0, \bar{\alpha}_{0}\right)$ and $\mathcal{D}_{\alpha_{0}}=\left\{x \in \bar{\Omega}: \phi(x)<\alpha_{0}\right\} \cup \mathcal{H}$. Then, $\partial \mathcal{D}_{\alpha_{0}}$ is a $C^{\infty}$ curve, since $\phi=\alpha_{0}$ and $\nabla \phi \neq 0$ on it. Moreover, $\overline{\mathcal{H}} \subset \mathcal{D}_{\alpha_{0}}$.

We will construct a subsolution $v$ in $\mathcal{I}_{\delta, T, \alpha_{0}}=\left\{(x, t) \in \mathcal{I}_{\delta, T}: x \in \Omega_{\alpha_{0}}\right\}$ where $\Omega_{\alpha_{0}}=\mathbb{R}^{2} \backslash \overline{\mathcal{D}_{\alpha_{0}}}$. As we observed in Section 3, if $u$ is the solution of $(\mathrm{P})$, then $u$ is continuous and, if $\delta>0$ is small enough and $T$ is big enough, then $u>0$ in the interior of $\mathcal{I}_{\delta, T, \alpha_{0}}$. Actually, $u>0$ also in the parabolic boundary of $\mathcal{I}_{\delta, T, \alpha_{0}}$.

We will construct a strict classical subsolution $v$ in $\mathcal{I}_{\delta, T, \alpha_{0}}$ such that, if $\delta>0$ is small enough and $T$ is large enough, there holds that $v<u$ in $\partial_{p} \mathcal{I}_{\delta, T, \alpha_{0}}$. Then, comparison will follow from Lemma 3.1.

As in the case of the supersolution, we construct $v$ as an approximation to $\tilde{V}(x, t)=G(x, t)\left(2 m \phi(x)(\ln t)^{-1}\right)^{1 / m}$. Indeed, we define

$$
v(x, t)=\eta \hat{c}(t) G(x, t) w(x, t),
$$

where

$$
\begin{aligned}
& 0<\eta<1, \hat{c}(t)=1-\kappa_{0}\left(\frac{T}{t}\right)^{\mu}, \text { with } \mu, \kappa_{0} \in(0,1), \text { and } \\
& w(x, t)=\left(\frac{2 m}{\log t}\right)^{\frac{\hat{\nu}(t)}{m}}\left((\phi(x))^{\hat{\nu}(t)}-\alpha_{0}^{\hat{\nu}(t)}\right)^{\frac{1}{m}}, \text { with } \alpha_{0} \in\left(0, \bar{\alpha}_{0}\right) \text { and } \\
& \hat{\nu}(t)=1+\frac{1}{\ln t} .
\end{aligned}
$$

Notice that $v$ vanishes on $\partial \Omega_{\alpha_{0}}$, and that $\Omega_{\alpha_{0}}$ approaches $\Omega$ as $\alpha_{0}$ goes to 0 . In order to check that $v$ is a subsolution, we will use the same kind of decomposition as the one we used for the supersolution in Section 3, namely $\partial_{t} v-\Delta v^{m}=\mathcal{A}+\mathcal{B}$, where

$$
\begin{aligned}
& \mathcal{A}=\eta \hat{c}^{\prime} G w+\eta \hat{c} w \partial_{t} G-\eta^{m} \hat{c}^{m} w^{m} \Delta G^{m}+\eta \hat{c} G \partial_{t} w, \\
& \mathcal{B}=-\eta^{m} \hat{c}^{m} G^{m} \Delta w^{m}-2 \eta^{m} \hat{c}^{m} \nabla w^{m} \cdot \nabla G^{m} .
\end{aligned}
$$

The next two lemmas are devoted to obtaining estimates for $\mathcal{A}$ and $\mathcal{B}$ good enough as to prove that $v$ is a subsolution in adequate sets. As in Lemmas 3.2 and 3.3, we have to consider separately two regions, the outer part of the inner region, which in this case is given by

$$
\mathcal{I}_{\delta, T, \alpha_{0}}^{\mathrm{O}}=\left\{(x, t) \in \mathcal{I}_{\delta, T, \alpha_{0}}:|x| \geq t^{\frac{1}{2 m}}(\ln t)^{-1}\right\},
$$

and the inner part of the inner region,

$$
\mathcal{I}_{\delta, T, \alpha_{0}}^{\mathrm{i}}=\left\{(x, t) \in \mathcal{I}_{\delta, T}:|x| \leq t^{\frac{1}{2 m}}(\ln t)^{-1}\right\} .
$$

As we will see, $\mathcal{A}$ is negative both in the outer and in the inner parts of the inner region. However, though $\mathcal{B}$ is negative in the inner part of the inner region, this is not necessarily the case in the outer part, and hence we have to check that, anyway, $\mathcal{A}+\mathcal{B}<0$ there.

Lemma 4.1. Let $\mathcal{A}$ be defined by (4.1)-(4.2) and $\delta_{*}$ as in Lemma 3.2. There are constants $\gamma=\gamma\left(m, M_{\phi}^{*}, \alpha_{0}\right)>0$ and $\mu_{*}=\mu_{*}\left(\eta, m, M_{\phi}^{*}, \kappa_{0}\right)>0$, and a time $T_{\mathcal{A}}=T_{\mathcal{A}}\left(\eta, m, M_{\phi}^{*}, \alpha_{0}\right)$ such that, for any $\delta \in\left(0, \delta_{*}\right), \mu \in\left(0, \mu_{*}\right)$, and $T \geq T_{\mathcal{A}}$,

$$
\mathcal{A}(x, t)<-\frac{\gamma \eta \hat{c}(t) w(x, t)}{t^{1+\frac{1}{m}}(\ln t)^{\frac{1}{m}}} \quad \text { in } \mathcal{I}_{\delta, T, \alpha_{0}} .
$$

Proof. A straightforward computation shows that

$$
\partial_{t} w(x, t)=\frac{w(x, t)}{m}\left(\frac{\ln \left(\frac{\ln t}{2 m}\right)}{t(\ln t)^{2}}-\frac{\hat{\nu}(t)}{t \ln t}-\frac{\ln \alpha_{0}}{t(\ln t)^{2}}-\frac{(\phi(x))^{\hat{\nu}(t)}\left(\ln \phi(x)-\ln \alpha_{0}\right)}{t(\ln t)^{2}\left((\phi(x))^{\hat{\nu}(t)}-\alpha_{0}^{\hat{\nu}(t)}\right)}\right) .
$$


Since $\hat{\nu}(t) \geq 1$, and $\phi(x)>\alpha_{0}$ in $\Omega_{\alpha_{0}}$, we conclude that there is a time $T_{m, \alpha_{0}}$, depending only on $m$ and $\alpha_{0}$ such that

$$
\partial_{t} w(x, t)<-\frac{w(x, t)}{2 m t \ln t} \quad \text { in } \mathcal{I}_{\delta, T, \alpha_{0}} \quad \text { if } T \geq T_{m, \alpha_{0}} .
$$

We next notice that

$$
t \hat{c}^{\prime}(t) \leq \mu^{\frac{1}{2}} \hat{c}(t) \quad \text { if } t \geq T \text { and } \mu^{\frac{1}{2}} \in\left(0, \frac{1-\kappa_{0}}{\kappa_{0}}\right) .
$$

Moreover, since $\phi(x) \leq \ln |x|+C$, there exists a time $T_{\eta, m}$, depending only on $\eta$ and $m$, such that for all $T \geq T_{\eta, m}$,

$$
(\eta w(x, t))^{m-1} \leq 1-\frac{1-\eta^{m-1}}{2} \text { in } \mathcal{I}_{\delta, T, \alpha_{0}} .
$$

We finally observe that there is a constant $\gamma_{*}=\gamma_{*}\left(m, M_{\phi}^{*}\right)>0$ such that

$$
\Delta G^{m}(x, t)<-\frac{\gamma_{*}}{t^{1+\frac{1}{m}}(\ln t)^{\frac{1}{m}}} \quad \text { in } I_{\delta, T} \text { if } \delta \in\left(0, \delta_{*}\right)
$$

see (3.5). Hence, since $\hat{c}(t)<1$ and $\partial_{t} G \leq \Delta G^{m}$, taking $T \geq \max \left(T_{m, \alpha_{0}}, T_{\eta, m}\right)$ we conclude that

$$
\begin{aligned}
\mathcal{A}(x, t) & <\eta \hat{c}(t) w(x, t)\left(\frac{\mu^{\frac{1}{2}} G(x, t)}{t}+\frac{\Delta G^{m}(x, t)\left(1-\eta^{m-1}\right)}{2}\right) \\
& \leq \frac{\eta \hat{c}(t) w(x, t)}{t^{1+\frac{1}{m}}(\ln t)^{\frac{1}{m}}}\left(\mu^{\frac{1}{2}} F_{*}(0)-\frac{\gamma_{*}\left(1-\eta^{m-1}\right)}{2}\right)
\end{aligned}
$$

from where the result follows just taking

$$
\mu_{*}=\left(\min \left(\frac{\gamma_{*}\left(1-\eta^{m-1}\right)}{4 F_{*}(0)}, \frac{1-\kappa_{0}}{\kappa_{0}}\right)\right)^{2} \quad \text { and } \quad \gamma=\frac{\gamma_{*}\left(1-\eta^{m-1}\right)}{4} .
$$

Lemma 4.2. Let $\mathcal{B}$ be defined by (4.1)-(4.2). There are a constant $\varpi=\varpi\left(m, M_{\phi}^{*}\right)$ and a time $T_{\mathcal{B}}^{\mathrm{o}}=T_{\mathcal{B}}^{\mathrm{o}}(\Omega, m)$ such that for all $\delta \in\left(0, \xi_{*}\right)$ and $T \geq T_{\mathcal{B}}^{\mathrm{o}}$,

$$
\mathcal{B}(x, t) \leq \frac{\varpi \eta \hat{c}(t) w(x, t)}{(t \ln t)^{1+\frac{1}{m}}} \quad \text { in } \mathcal{I}_{\delta, T, \alpha_{0}}^{\mathrm{o}} .
$$

Moreover, there is a time $T_{\mathcal{B}}^{\mathrm{i}}=T_{\mathcal{B}}^{\mathrm{i}}\left(\Omega, m, M_{\phi}^{*}\right)$ such that $\mathcal{B} \leq 0$ in $\mathcal{I}_{\delta, T, \alpha_{0}}^{\mathrm{i}}$ for all $T \geq T_{\mathcal{B}}^{\mathrm{i}}$ and $\delta \in\left(0, \xi_{*}\right)$.

Proof. We perform computations similar to those in Lemma 3.3 that lead to (3.8), with $w$ is as in (4.1). Observe that $\Delta w^{m} \geq 0$, since $\hat{\nu}(t) \geq 1$. Moreover, since $|x||\nabla \phi(x)| \leq C$ in $\bar{\Omega}$, there is a constant $\varpi_{1}=\varpi_{1}\left(m, M_{\phi}^{*}\right)$ such that

$$
-2\left(\nabla G^{m} \cdot \nabla w^{m}\right)(x, t) \leq \frac{\varpi_{1}}{t^{1+\frac{1}{m}}(\ln t)^{\frac{1}{m}} \phi(x)}\left(\frac{2 m \phi(x)}{\ln t}\right)^{\hat{\nu}(t)} \quad \text { in } \mathcal{I}_{\delta, T, \alpha_{0}} .
$$

On the other hand, since $\phi(x) \geq \ln |x|-C$, there is a time $T_{\phi}^{\circ}=T_{\phi}^{\mathrm{o}}(\Omega, m)$ such that $\phi(x) \geq(\ln t) /(4 m)$ in $\mathcal{I}_{\delta, T, \alpha_{0}}^{\mathrm{o}}$ if $T \geq T_{\phi}^{\mathrm{o}}$. In particular, there is a large enough time $T_{\mathcal{B}}^{0}=T_{\mathcal{B}}^{0}(\Omega, m) \geq T_{\phi}^{\mathrm{o}}$ such that

$$
w(x, t) \geq\left(\frac{1}{2}\right)^{\frac{\hat{\nu}(t)}{m}}\left(\frac{2 m \phi(x)}{\ln t}\right)^{\frac{\hat{\nu}(t)}{m}} \geq \frac{1}{2}\left(\frac{2 m \phi(x)}{\ln t}\right)^{\hat{\nu}(t)} \quad \text { in } \mathcal{I}_{\delta, T, \alpha_{0}}^{\mathrm{o}} \text { if } T \geq T_{\mathcal{B}}^{0}
$$

and (4.4) follows easily with $\varpi=8 m \varpi_{1}$, since $c(t), \eta \leq 1$.

As for the inner part of the inner region, we observe that there is a time $T_{\phi}=$ $T_{\phi}\left(\Omega, m, M_{\phi}^{*}\right)$ such that $\phi(x) \leq(\ln t) / m$ in $\mathcal{I}_{\delta, T, \alpha_{0}}$, and hence this inequality also 
holds in $\mathcal{I}_{\delta, T, \alpha_{0}}^{\mathrm{i}}$, for $T \geq T_{\phi}$ and $\delta \in\left(0, \xi_{*}\right)$. Therefore, since $|\nabla \phi(x)|$ is comparable to $1 /|x|$ in $\Omega$, there is a constant $\varpi_{2}$ such that

$$
-\left(G^{m} \Delta w^{m}\right)(x, t) \leq-\frac{\varpi_{2}}{t^{1+\frac{1}{m}} \phi(x)}\left(\frac{2 m \phi(x)}{\ln t}\right)^{\hat{\nu}(t)} \quad \text { in } \mathcal{I}_{\delta, T, \alpha_{0}}^{\mathrm{i}},
$$

that combined with $(4.5)$ yields $\mathcal{B} \leq 0$ if $T \geq T_{\mathcal{B}}^{\mathrm{o}} \geq T_{\phi}$, for some $T_{\mathcal{B}}^{\mathrm{o}}=T_{\mathcal{B}}^{\mathrm{o}}\left(\Omega, m, M_{\phi}^{*}\right)$ large enough.

Combining the estimates in Lemmas 4.1 and 4.2, we immediately get that $v$ is a strict classical subsolution of the equation.

Corollary 4.1. Let $v$ be defined by (4.1) and $\delta_{*}$ and $\mu_{*}$ as in Lemma 4.3. There is a time $T_{*}=T_{*}\left(\eta, \delta, \Omega, M_{\phi}^{*}, \alpha_{0}\right)$ such that

$$
\partial_{t} v-\Delta v^{m}<0 \quad \text { in } \mathcal{I}_{\delta, T, \alpha_{0}} \text { for all } \delta \in\left(0, \delta_{*}\right), \mu \in\left(0, \mu_{*}\right) \text {, and } T \geq T_{*} .
$$

In order to prove that $u>v$ in $I_{\delta, T, \alpha_{0}}$, it is then just enough to prove that this inequality holds on the parabolic boundary of this set. The ordering in the outer boundary will come from the far-field behavior. It is here where we will be performing the matching.

Proposition 4.1. Let $u$ be a weak solution to $(\mathrm{P}), 0<\eta<1, v$ as in (4.1), and $\delta_{*}$ and $\mu_{*}$ as in Lemma 4.1. Given $\alpha_{0} \in\left(0, \bar{\alpha}_{0}\right)$ and $\delta \in\left(0, \delta_{*}\right)$, there is a time $T^{-}=T^{-}\left(\eta, \delta, \Omega, m, M_{\phi}^{*}, \alpha_{0}\right)>0$ such that for any $T \geq T^{-}$there is a value $\kappa_{0}>0$ such that, for all $\mu \in\left(0, \mu_{*}\right)$, there holds that $u>v$ in $\mathcal{I}_{\delta, T, \alpha_{0}}$.

Proof. We first note that $v=0<u$ on $\partial \Omega_{\alpha_{0}} \times \mathbb{R}_{+}$. As for the outer boundary, we have from (1.10) that there exists a time $T_{\eta, \delta, m}>0$ such that, for $|x|=$ $\delta t^{1 /(2 m)}(\ln t)^{-(m-1)(2 m)}, t \geq T_{\Omega, \eta, \delta}$,

$$
u(x, t) \geq G(x, t)-\frac{(1-\eta) F_{*}\left(\delta_{*}\right)}{2(t \ln t)^{\frac{1}{m}}} \geq\left(1-\frac{1-\eta}{2}\right) G(x, t) .
$$

On the other hand, there is a time $T_{\Omega, \eta, \delta}>0$ such that,

$$
\eta w(x, t) \leq 1-\frac{1-\eta}{2} \quad \text { for }|x|=\delta t^{\frac{1}{2 m}}(\ln t)^{-\frac{m-1}{2 m}}, \text { with } t \geq T_{\eta, \delta, m} .
$$

Since $\hat{c}(t)<1$, we conclude that

$$
u(x, t)>v(x, t) \quad \text { for }|x|=\delta t^{\frac{1}{2 m}}(\ln t)^{-\frac{m-1}{2 m}} \text {, with } t \geq \max \left(T_{\eta, \delta, m}, T_{\Omega, \eta, \delta}\right) .
$$

Moreover, by (1.11), given $\delta \in\left(0, \xi_{*}\right)$, there is a time $T_{\xi_{*}}=T_{\xi_{*}}\left(\delta, \Omega, m, \alpha_{0}\right)$ such that for some $\ell>0$, there holds that $u(x, T) \geq \ell$ if $(x, T) \in \overline{\mathcal{I}_{\delta, T, \alpha_{0}}}, T \geq T_{\xi_{*}}$. Thus, given $T \geq T^{-}:=\max \left(T_{*}, T_{\eta, \delta, m}, T_{\Omega, \eta, \delta}, T_{\xi_{*}}\right)$, where $T_{*}$ is the time given by Corollary 4.1,

$$
v(x, T)<\frac{\left(1-\kappa_{0}\right)(1+\eta) F_{*}(0)}{2(T \ln T)^{\frac{1}{m}}} \leq \ell \leq u(x, T) \quad \text { in } \mathcal{I}_{\delta, T, \alpha_{0}},
$$

if $\kappa_{0} \in(0,1)$ is close enough to 1 . The result follows from Lemma 3.1.

The "lower part" of Theorem 1.1 is now easy.

Theorem 4.1. Assume the hypotheses of Theorem 1.1 and let $\mathcal{M}$ be as in Theorem 3.1. Given $\varepsilon>0$, there exists a time $T_{\varepsilon}$ such that $\mathcal{M} \geq-\varepsilon$ in $\mathcal{I}_{\delta, T_{\varepsilon}}$. 
Proof. Given $\varepsilon>0$ small, we take $\alpha_{0}=\varepsilon^{m}(2 m)^{-1}\left(F_{*}(0)\right)^{-m}$. Then,

$$
\frac{t^{\frac{1}{m}}(\ln t)^{\frac{2}{m}}}{(\ln (|x|+\mathrm{e}))^{\frac{1}{m}}}\left(\frac{2 m \phi(x)}{\ln t}\right)^{\frac{1}{m}} G(x, t) \leq \varepsilon \quad \text { in }\left(\Omega \backslash \Omega_{\alpha_{0}}\right) \times \mathbb{R}_{+} .
$$

Hence, it is enough to prove that there exists a time $T_{\varepsilon}$ such that $\mathcal{M} \geq-\varepsilon$ in $\mathcal{I}_{\delta, T_{\varepsilon}, \alpha_{0}}$. To this aim, we use the decomposition (3.9), with $c, W$ and $\nu$ substituted respectively by $\hat{c}, w$ and $\hat{\nu}$.

Let $\eta=1-\varepsilon$ and $\delta \in\left(0, \delta_{*}\right)$. By Proposition 4.1, given any $T \geq T^{-}$, there are values $\kappa_{0}>0$ and $\mu \in\left(0, \mu_{*}\right)$ such that

$$
u(x, t)-\hat{c}(t) w(x, t) G(x, t) \geq-\varepsilon \hat{c}(t) w(x, t) G(x, t) \quad \text { if }(x, t) \in \mathcal{I}_{\delta, T, \alpha_{0}} .
$$

On the other hand, since $(a-b)^{1 / m}-a^{1 / m} \geq-b^{1 / m}$ for all $a \geq b>0$ if $m>1$, we have

$$
w(x, t)-\left(\frac{2 m \phi(x)}{\ln t}\right)^{\frac{\hat{\nu}(t)}{m}} \geq-(\ln t)^{-\frac{\hat{\nu}(t)}{m}}\left(\frac{\varepsilon}{F_{*}(0)}\right)^{\hat{\nu}(t)} \geq-K \varepsilon
$$

for some constant $K$ independent of $\varepsilon$, assuming, without loss of generality, $T \geq \mathrm{e}$ and $\varepsilon \leq 1$.

We conclude as in the proof of Theorem 3.1.

\section{REFERENCES}

[1] Barenblatt, G. I. On some unsteady motions of a liquid and gas in a porous medium. (Russian) Akad. Nauk SSSR. Prikl. Mat. Meh. 16 (1952), no. 1, 67-78.

[2] Barenblatt, G. I.; Zel'dovic, Ya. B. On dipole solutions in problems of non-stationary filtration of gas under polytropic regime. (Russian) Prikl. Mat. Mekh. 21 (1957), no. 5, 718-720.

[3] Bénilan, Ph. The Laplace operator. In: Dautray, R.; Lions, J.-L. (eds.) "Mathematical analysis and numerical methods for science and technology. Vol. 1. Physical origins and classical methods." pp. 220-666. Springer-Verlag, Berlin, 1990. ISBN: 3-540-50207-6; 3-540-66097-6.

[4] Brändle, C.; Quirós, F.; Vázquez, J.L. Asymptotic behaviour of the porous media equation in domains with holes. Interfaces Free Bound. 9 (2007), no. 2, 211-232.

[5] Caffarelli, L. A.; Friedman, A. Continuity of the density of a gas flow in a porous medium. Trans. Amer. Math. Soc. 252 (1979), 99-113.

[6] Cortázar, C.; Elgueta, M.; Quirós, F.; Wolanski, N. Asymptotic behavior for a nonlocal diffusion equation on the half line. Discrete Contin. Dyn. Syst. 35 (2015), no. 4, 1391-1407.

[7] Cortázar, C.; Elgueta, M.; Quirós, F.; Wolanski, N. Asymptotic behavior for a nonlocal diffusion equation in exterior domains: The critical two-dimensional case. J. Math. Anal. Appl. 436 (2016), no. 1, 586-610.

[8] Cortázar, C.; Quirós, F.; Wolanski, N. Near field asymptotic behavior for the porous medium equation on the half-line. Adv. Nonlinear Stud. 17 (2017), no. 2, 245-254.

[9] Friedman, A.; Kamin, S. The asymptotic behavior of gas in an n-dimensional porous medium. Trans. Amer. Math. Soc. 262 (1980), no. 2, 551-563.

[10] Gilding, B. H.; Goncerzewicz, J. Large-time behaviour of solutions of the exterior-domain Cauchy-Dirichlet problem for the porous media equation with homogeneous boundary data. Monatsh. Math. 150 (2007), no. 1, 11-39.

[11] Herraiz, L. Asymptotic behaviour of solutions of some semilinear parabolic problems. Ann. Inst. H. Poincaré Anal. Non Linéaire 16 (1999), no. 1, 49-105.

[12] Kamin, S.; Vázquez, J. L. Asymptotic behaviour of solutions of the porous medium equation with changing sign. SIAM J. Math. Anal. 22 (1991), no. 1, 34-45.

[13] King, J. R. Integral results for nonlinear diffusion equations. J. Engrg. Math. 25 (1991), no. 2, 191-205.

[14] Pattle, R. E. Diffusion from an instantaneous point source with a concentration-dependent coefficient. Quart. J. Mech. Appl. Math. 12 (1959), 407-409.

[15] Vázquez, J.L. Asymptotic beahviour for the porous medium equation posed in the whole space. J. Evol. Equ. 3 (2003), no. 1, 67-118.

[16] Vázquez, J. L. "The porous medium equation. Mathematical theory". Oxford Mathematical Monographs. The Clarendon Press, Oxford University Press, Oxford, 2007. ISBN: 978-0-19856903-9. 
[17] Zel'dovič, Ya. B.; Kompaneets, A.S. On the theory of propagation of heat with the heat conductivity depending upon the temperature. Collection in honor of the seventieth birthday of academician A. F. Ioffe, pp.61-71. Izdat. Akad. Nauk SSSR, Moscow, 1950.

[18] Ziemer, W. P. Interior and boundary continuity of weak solutions of degenerate parabolic equations. Trans. Amer. Math. Soc. 271 (1982), no. 2, 733-748.

CARMEN CORTÁzAR

Departamento de Matemática, Pontificia Universidad Católica de Chile Santiago, Chile.

E-mail address: ccortaza@mat.puc.cl

FERNANDO QUIRÓs

Departamento de Matemáticas, Universidad Autónoma de Madrid 28049-MADRID, SPAIN.

E-mail address: fernando.quiros@uam.es

NOEMÍ WOLANSKI

Departamento de Matemática, FCEyn, Universidad de Buenos Aires, AND IMAS, CONICET,

(1428) Buenos Aires, Argentina.

E-mail address: wolanski@dm.uba.ar 